\title{
Influence of Dental Treatment on Outcome of Graves' Disease Treated with Anti-Thyroid Drug
}

\section{Toshihide Yamamoto*}

Yao Tokushukai General Hospital, Yao, Osaka, Japan

*Corresponding author: Toshihide Yamamoto, MD, Yao Tokushukai General Hospital, 1-17, Wakakusa-cho, Osaka 581-0011 Japan, Tel: 8172-93-8500; E-mail: toshihide.yamamoto@tokushukai.jp

Received: 18 Feb, 2021 | Accepted: 08 Mar, 2021 | Published: 12 Mar, 2021

Citation: Yamamoto T (2021) Influence of Dental Treatment on Outcome of Graves' disease Treated with Anti-Thyroid Drug. Int J Endocrinol Metab Disord 7(1): dx.doi.org/10.16966/2380-548X.173

Copyright: (c) 2021 Yamamoto T. This is an open-access article distributed under the terms of the Creative Commons Attribution License, which permits unrestricted use, distribution, and reproduction in any medium, provided the original author and source are credited.

\section{Abstract}

Purpose: The relations between Time Courses (TCS) of anti-TSH receptor auto-antibodies (TRAb) during Anti-Thyroid Drug (ATD) treatment and the outcome of Graves' Disease (GD) were sought in relation to dental treatment.

Methods: In one year period, there were $61 \mathrm{GD}$ patients being treated with ATD in a titration regimen. Their TRAb levels were measured by a $2^{\text {nd }}$ generation assay (designated as hTRAb) periodically. Forty-nine patients were selected whose records contained dental findings and endocrine data before ATD treatment. They were divided into four groups depending on the number of teeth with metal crown placement (henceforth, wMC); no tooth wMC (group A), one or two teeth wMC (group B), three or four teeth wMC (group C), and five or more teethwMC (group D). The relation of numbers of teeth wMC and goiter size, free thyroxine, hTRAb (50 patients) and TCs (36 patients) were sought.

Results: Thyroid gland size increased with numbers of teeth wMC. The hTRAb levels before ATD of patients having $\leq 2$ teeth wMC (groups A and $B$ ) were lower than those of patients having more than $\geq 5$ teeth wMC (group D). Most TCs of groups $A$ and B were located in the lower region and became undetectable or in gray zone in 18 months while TCs of groups $C$ and D scattered in the higher region and declined more slowly.

Conclusion: Teeth wMC without endodontic treatment are conceivably complicated by radicular abscess in some of them, which appears to be associated with sustained TRAb production.

Keywords: Graves' disease; Anti-thyroid drug; Periodontal disease; Anti-TSH receptor antibodies

\section{Background}

Anti-thyroid drugs (ATDs) have been employed for treatment of Graves' disease (GD) worldwide. However, remission of GD through ATD treatment is still unsatisfactory, eg., remission rates after ATD withdrawal reported in two recent meta-analyses are $48.7 \%$ [1] and $57 \%$ [2]. TSH receptor-specific treatment remains in investigational stage [3]. Means to improve remission rate with currently available ATD are worthy of being sought. Graves' disease is known as an autoimmune disease caused by TSH-receptor-stimulating immunoglobulin $[4,5]$. Provided the production of TSH-receptorstimulating immunoglobulin antibodies is affected by comorbid disorder, the outcome of drug-treated GD might be improved by treating or eliminating comorbid disease.

GD patients were treated with ATD while serum anti-TSH receptor auto-antibodies (TRAb) levels were periodically measured. The time courses (TCs) of TRAb were drawn and the relations between the TC patterns and the outcomes of GD were investigated. It was found that remission from GD was associated with two patterns, a smoothly declining pattern and a smoothly declining pattern with one or two spikes in TRAb levels in the periods of a flare-up of hyperthyroidism. Hence, a possibility was raised that the spikes could be used as a clue to investigate comorbid illness associated with the flair-up of GD [6].

When examining two GD patients at clinic who had been treated with ATD for years because of persistently high TRAb levels, they were observed to have many teeth with metal crown placement (henceforth, abbreviated as metal crown) as well as a few residual roots of broken teeth (henceforth, abbreviated as residual roots). Influences of comorbid disorders on GD are observed in inflammatory disorders, seasonal allergic rhinitis [7] and Helicobacter pylori infection [8]. It was thought that chronic dental root(s) inflammation might influence immune dysregulation of GD.

In this study, relations of teeth with metal crown placement with pre-treatment TRAb levels and changes of TRAb levels during ATD treatment are evaluated in GD patients with teeth metal crown treatment in 1-5 or more teeth or without dental caries(or edentulous). 


\section{Patients and Treatment of GD}

In one-year period from July 2019 till June 2020, 61 GD patients were treated with ATD at the endocrine clinic of Yao Tokushukai General Hospital. All patients were evaluated and treated by the author. ATD treatment had started before this period in 50 patients and the treatment was started in this period in 11 patients. From 61 patients, 12 patients were excluded for following reasons; no description of dental condition in the medical records (three patients), absence of endocrine data before TRAb treatment because ATD being started elsewhere (eight patients), written informed consent to publishing data denied (one patient).

A diagnosis of GD was based on clinical manifestations, serum thyroid and thyrotrophic hormones, and TRAb. The thyroid gland was palpated by both hands from behind patients. The size of the thyroid was classified as follows: grade I, thyroid felt beneath the author's index and middle fingers (approximately $4 \mathrm{~cm}$ ) or less, grade II, felt greater than two-finger breadth, grade III, visible without neck hyperextension. Patients were treated with either thiamazole or propylthiouracil in a titration regimen. Forty-nine patients who had provided informed consent were divided into four groups: Group A (15 patients), either dental caries-free, small metal inlay placement in a few teeth, or edentulous; Group B (11 patients), one or two teeth with metal crown; group C (12 patients), three or four teeth with metal crown; and group D (11 patients), five or more teeth treated with metal crown. A few patients of groups $C$ and $D$ had residual roots as well. The demographic data and goiter sizes at the beginning of ATD treatment are summarized in table 1 .

TRAb serum levels were measured in a second-generation competitive binding assay with DYNO test TRAK ${ }^{\mathrm{TM}}$ humankit [9] (manufactured by BRAHMS AG, Berlin). The assay was performed by Biomedical Laboratories (BML, Matoba, Saitama).TRAb measured by DYNO test is hence abbreviated as hTRAb. hTRAb level of $<1.0$ IU/L was considered to be negative (GD denied) and levels between 1.0 and $1.5 \mathrm{IU} / \mathrm{L}$ as gray zone level [9]. Free thyroxine (T4) levels were measured in a competitive binding assay kit (FT4 Abbott TM, Abbott Japan, Ciba, Japan).

The hTRAb levels were measured every 3 or 4 months in patients with declining titers and less frequently in patients with high or slowly decreasing hTRAb levels. ATD was withheld in two conditions: first, ATD was continued until TRAb levels had been undetectable consecutively twice, separated by 3 or more months; second, thyroid function tests had been within the reference range and hTRAb levels remained in the gray zone $(1.0 \sim 1.5 \mathrm{IU} / \mathrm{L})$ while being maintained on a minimal dose (one tablet every other day) of ATD for 6 or more months. If the dosage of ATD could be reduced to minimal dose, ATD was continued even if hTRAb levels remained persistently in gray zone. Thirty-six patients had four or more serial data of hTRAb levels enough to draw the time courses of hTRAb, which were are drawn in diagrams with time (month) in the abscissa and hTRAb levels (in logarithmic scale) in the ordinate.

\section{Results}

Demographic data and thyroid sizes of the forty-nine patients are summarized in table 1 . The size of the thyroid gland appears to increase with numbers of teeth with metal crown, i.e., $(\mathrm{p}<0.05,3 \times 4$ contingency table).

The levels of free thyroxine and hTRAb levels in the start of ATD are shown in figure 1 . The free T4 levels were not different between any two groups (Mann-Whitney U test, $\mathrm{P}>0.05$ ) (Figure 1, panel A).
The hTRAb levels were lower in group A and B than those of group $\mathrm{D}$, i.e., group $\mathrm{A}$ vis group $\mathrm{D}, \mathrm{P}<0.01$ and group $\mathrm{B}$ vis group $\mathrm{D}, \mathrm{p}<0.05$ (Mann-Whitney U Test) (figure 1, panel B). The hTRAb levels were not different between other two groups.

The TCs of 36 patients are illustrated in four panels (TCs of group $\mathrm{A}$ in panel A, and so on) of figure 2. The TCs of the other 13 patients are not drawn because their serial hTRAb levels were measured in periods not long enough to assess TC patterns. The distribution of TCs of groups A and B and that of groups C and D are dissimilar, i.e., most TCs of groups A and B were located more or less closely in the lower region (figure 2, panels A and B) while the TCs of groups $\mathrm{C}$ and $\mathrm{D}$ were scattered in the higher region (figure 2, panels $\mathrm{C}$ and D. The hTRAb levels of eight patients of group A were progressively reduced to undetectable levels twice consecutively within six months (four patients) or converged in the gray zone in 12 months (four patients); the levels of six patients of group B decreased slower than those of group A and converged in the gray zone within 18 months. The reduction of hTRAb levels were much slower in groups C and D, i.e., the levels converged in gray zone within 18 months in two patients of group C and one patient of group D. The levels of hTRAb remained in gray zone after six months' ATD administration in six patients of groups A and B. In three of them, ATD was discontinued after minimal dose of it having been administered for six or more months.

A few of patients of groups $C$ and D needed occasional dental treatment while being treated with ATD. Three patients of group A and one of group B achieved remission defined by absence of relapse within one year after withdrawal of ATD.

\section{Discussion}

In the present observation, the administration of ATD was conducted according to hTRAb levels as if the levels were a surrogate biomarker of immune dysregulation. There are a few caveats to this assumption: the assay employed is a $2^{\text {nd }}$ generation competitive binding assay which cannot distinguish stimulation and blocking antibodies; some patients with GD reportedly develop hyperthyroidism after ATD withdrawal despite negative hTRAb [10]. As the hTRAb levels of most patients of groups A and B correlate well with their clinical courses, their hTRAb levels are considered to consist mainly of TSH receptor stimulating antibodies. The thyroid function could not be

Table 1: Demographic and clinical data.

\begin{tabular}{|c|c|c|c|c|c|c|}
\hline & \multirow{2}{*}{ Gender } & \multirow{2}{*}{ Number } & \multirow{2}{*}{$\begin{array}{l}\text { Age, Range, } \\
\text { Median }\end{array}$} & \multicolumn{3}{|c|}{ Goiter size* } \\
\hline & & & & 1 & II & III \\
\hline \multirow{2}{*}{$\begin{array}{l}\text { Group A (no } \\
\text { metal crown or } \\
\text { edentulous) }\end{array}$} & Women & 14 & $10-65,44.5$ & \multirow{2}{*}{8} & \multirow{2}{*}{5} & \multirow{2}{*}{2} \\
\hline & Men & 1 & 22 & & & \\
\hline \multirow{2}{*}{$\begin{array}{l}\text { Group B (metal } \\
\text { crown, one or two } \\
\text { teeth) }\end{array}$} & Women & 7 & $29-53,45$ & \multirow{2}{*}{2} & \multirow{2}{*}{8} & \multirow{2}{*}{1} \\
\hline & Men & 4 & $30-53,39.5$ & & & \\
\hline \multirow{2}{*}{$\begin{array}{l}\text { Group C (metal } \\
\text { crown, three or } \\
\text { four teeth) }\end{array}$} & Women & 10 & $25-66,44.5$ & \multirow{2}{*}{3} & \multirow{2}{*}{6} & \multirow{2}{*}{3} \\
\hline & Men & 2 & 38,45 & & & \\
\hline \multirow{2}{*}{$\begin{array}{l}\text { Group D (metal } \\
\text { crown, five or } \\
\text { more teeth) }\end{array}$} & Women & 8 & $37-62,49.5$ & \multirow{2}{*}{3} & \multirow{2}{*}{2} & \multirow{2}{*}{6} \\
\hline & Men & 3 & $45,61,64$ & & & \\
\hline
\end{tabular}

*I, palpable under index and middle fingers, II, bigger than two-finger breadth, III, visible without neck Hyperextension. Size of goiter increases with number of teeth with metal crown placement $(p<0.05,3 \times 4$ contingency table), at start of anti-thyroid drug administration. 

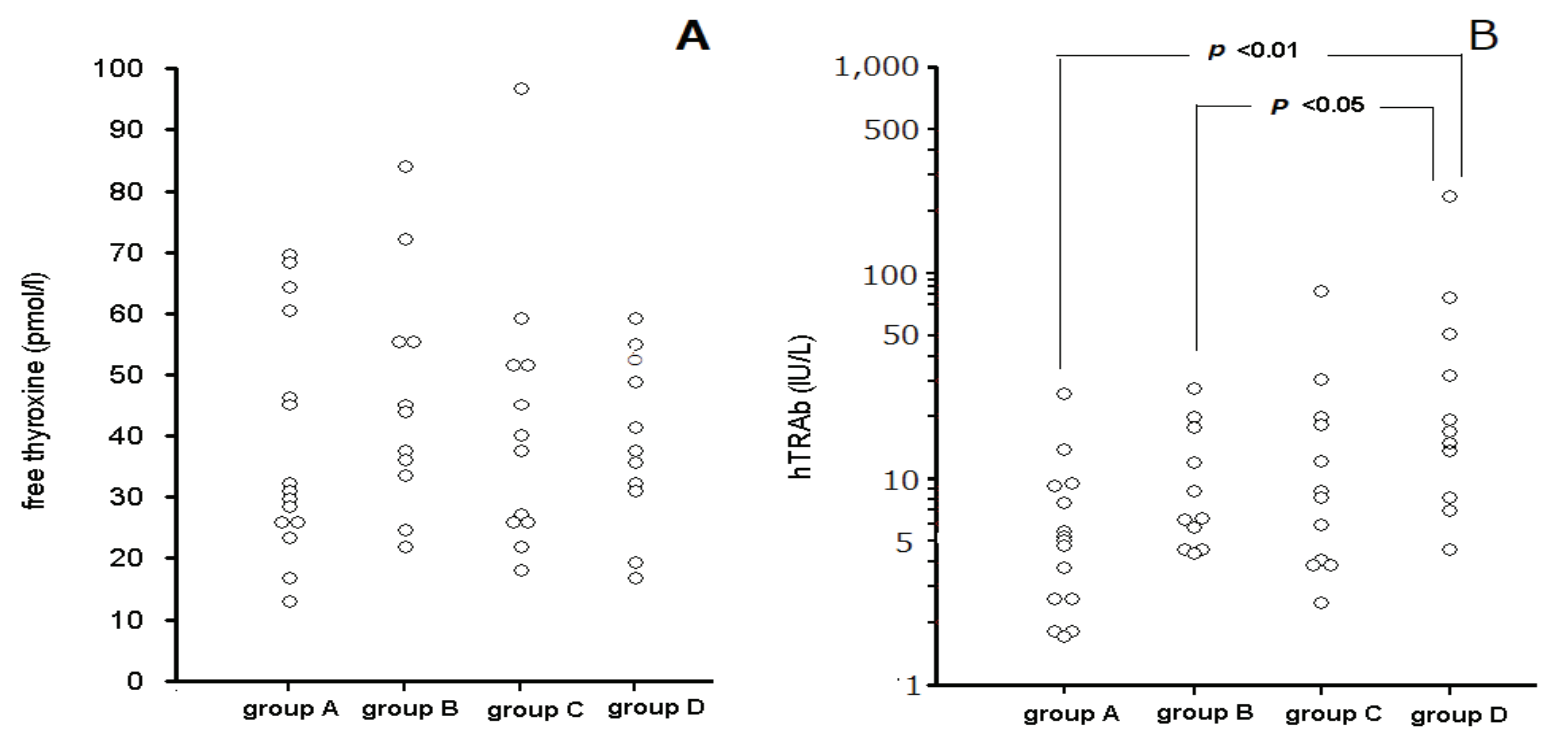

Figure 1: Comparison of free thyroxine and anti-TSH receptor antibodies before initiation of anti-thyroid drug administration in four groups. Panel A shows free thyroxine levels of four groups. Panel B shows anti-TSH receptor antibody levels of four groups. The anti-human-type thyroid receptor antibody is designated as hTRAb.
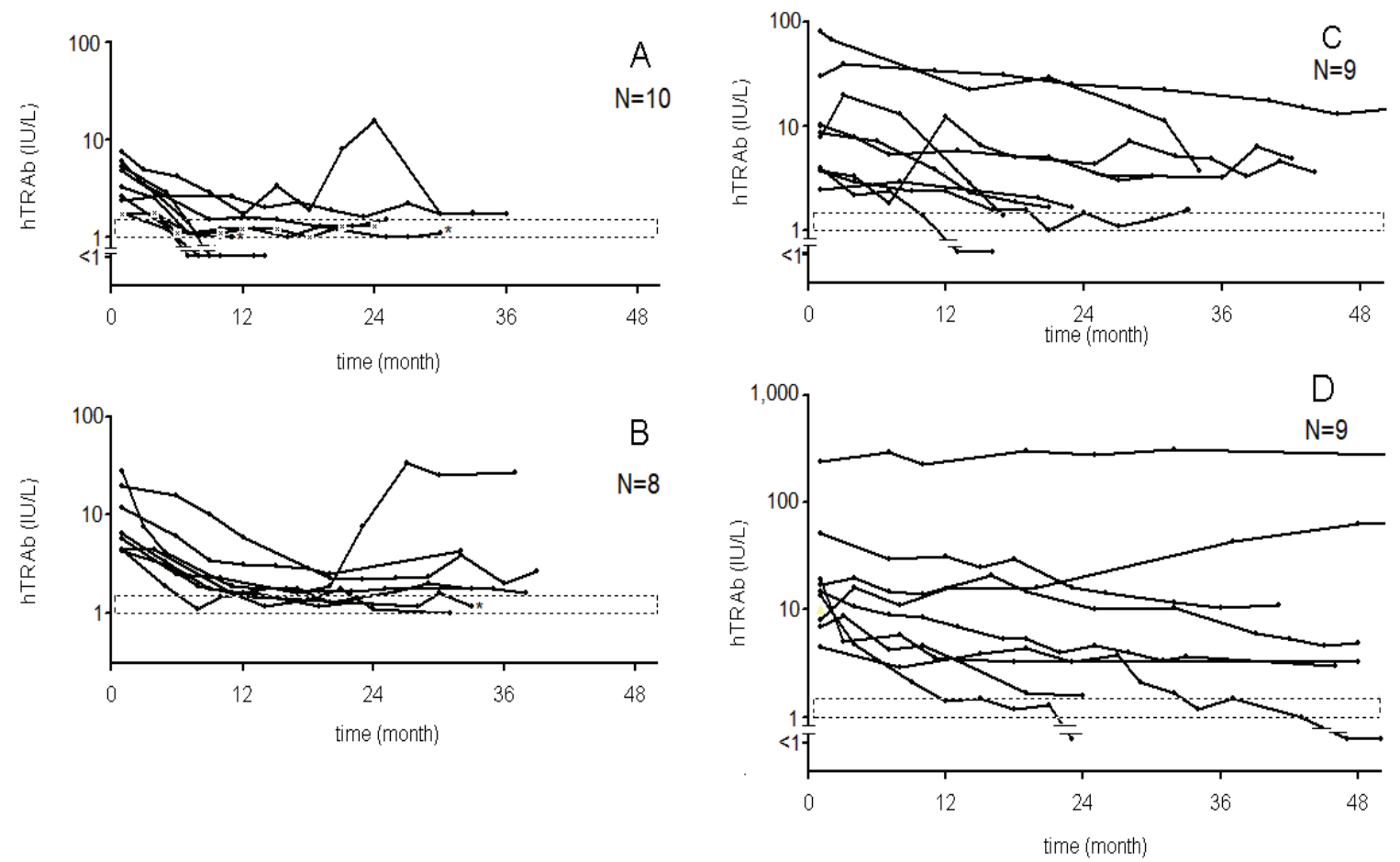

Figure 2: Time courses of anti-TSH receptor antibodies during anti-thyroid drug treatment.

The hTRAb data are log-transformed. Panel A shows time courses (TCS) of patients without dental caries (marked by bold circles) and those of one edentulous patient (marked by X). Panel B shows TCs of patients with metal crown placement in one or two teeth. Panel C shows TCs of patients with metal crown placement in three or four teeth. Panel D shows TCs of patients with metal crown placement in five or more teeth. Gray zones of hTRAb (1.0-1.5 IU/L) are shown by rectangular bands enclosed by dotted lines (panels A, B, C, and D). In panels A and B, TCs of three patients whose ATD was discontinued because of hTRAb remaining in gray zone are shown by asterisks at the right end of TCs. 
not determined primarily by gray-zone hTRAb levels, i.e., ATD was discontinued in three patients of groups A and B because of hTRAb levels remaining in gray zone while being treated with minimal dose of ATD. ATD could not be discontinued in other three patients despite hTRAb levels remaining in gray zone for failure to reduce ATD to minimal dose.

The present results are summarized as follows: high incidence of grade III goiter in group D patients, higher pre-treatment hTRAb levels of group D patients, slow declining TC slopes of patients of groups $\mathrm{B}$, and much slower declining TC slopes of groups $\mathrm{C}$ and $\mathrm{D}$. These findings suggest teeth with metal crown are causally related to sustained production of TRAb. Accurate assessment of the dental alveoli of metal-capped teeth cannot be made by non-dentist endocrinologists. Teeth with metal crown placement, however, is recognized and counted.

In Japan, almost every patient receives dental care under national health insurance. As the cost of endodontic treatment is not covered by the national insurance, most patients choose pulpotomy without endodontic treatment*. Pulpotomy without endodontic treatment often results in chronic apical abscess [11]. As metal crown is placed after pulpotomy, chronic radicular abscess is presumably present in some of the teeth with metal crown. The long-term systemic harmful effects of asymptomatic radicular abscess have not been known in medical world.

Accurate assessment of the dental alveoli of metal-capped teeth cannot be made by non-dentist endocrinologists. In the present study, the number of teeth with metal crown placement was counted which is easily recognizable and countable. Though the dental alveoli of teeth with metal crown had not been examined radiographically in every patient in this study, radicular abscess was thought to be present in some of the teeth with metal crown of patients of groups $\mathrm{B}, \mathrm{C}$, and D. Patients having more than three teeth with metal crown likely had chronic inflammation in the periodontium of some of the pulpotomized teeth. Though the dental condition did not interfere with mastication and remained asymptomatic except for an occasional toothache in these patients, chronic inflammation around the roots of pulpotomized teeth supposedly influences immune dysregulation of GD. The present finding remains to be proved in more patients in order to improve remission rate of GD by careful management of periodontal inflammation.

\section{Conclusion}

Most patients, either dental caries-free or edentulous, obtained remission from GD within 18 months of ATD treatment while patients having three or more teeth treated with metal crown as well as residual roots had persistently high levels of TRAb and required prolonged ATD treatment. Some of the pulpotomized teeth are likely complicated by chronic radicular abscess, which supposedly influences autoimmunity of GD.

${ }^{\star}$ The national insurance cost for pulpotomy without endodontic treatment is set about $¥ 6,000$ per root (nearly equivalent of $\$ 57.00$ ). Patients have to pay 10 to $30 \%$ of this cost depending on their taxable incomes. As endodontic treatment cost is about $¥ 100,000$ (roughly $\$ 943$ ) per root outside the insurance, very few patients prefer endodontic treatment.

\section{Acknowledgements}

The author is thankful for advice of Haruhiko Kanoh D.D.S. The author has treated patients with Graves' disease under the health insurance without any experimental intervention. The publication of this observation study was approved by Medical Ethics Committee of Yao Tokushukai General Hospital in September, 2020 (Chairman, H.Ishihara, M.D., document \# 2020-03).

\section{Conflicts of Interest}

The author has no conflicts of interest to declare.

\section{References}

1. Struja T, Fehlberg H, Kutz A, Guebelin L, Degen C, et al. (2017) Can we predict relapse in Graves' disease? Results from a systematic review and meta-analysis. Eur J Endocrinol 176: 87-97.

2. Azzizi F, Malboosbat R (2017) Long-term antithyroid drug treatment: A systemic review and meta-analysis. Thyroid 27: 1223-1231.

3. Lane LC, Cheetham TD, Perros P, Pearce SHS (2020) New therapeutic horizons for Graves' hyperthyroidism. Endocr Rev 41: 873-884.

4. Kriss JP, Pleshakov V, Chien JR (1964) Isolation and identification of the long-acting thyroid stimulator and its relation to hyperthyroidism and circumscribed pretibial myxedema. J Clin Endocrinol Metab 24: 1005-1028.

5. Weetman AP, Yateman ME, Ealey PA, black CM, Reimer CB, et al. (1990) Thyroid-stimulating antibody activity between different immunoglobulin G subclasses. J Clin Invest 86: 723-727.

6. Yamamoto T (2019) Outcome of Graves' disease treated with antithyroid drugs and time courses of anti-TSH receptor antibodies. Int J Endocrinol Metab Disord 5: 1.

7. Hidaka Y, Amino N, Iwatani Y, Itoh E, Matsunaga M, et al. (1993) Recurrence of thyrotoxicosis after attack of allergic rhinitis in patients with Graves' disease. J Clin Endocrinol Metab 77: 16671670.

8. Bassi V, Sentinelli C, Lengo A, Romano C (2010) Identification of a correlation between Helicobacter pylori infection and Graves' disease. Helicobacter 15: 558-562.

9. Costagliola S, Morgenthaler NG, Hoermann R, Badenhoop K, Struck $J$, et al. (1999) Second generation assay for thyrotropin receptor antibodies has superior diagnostic sensitivity for Graves' disease. J Clin Endocrinol Metab 84: 90-97.

10. Kubota S, Tamai H, Ohye H, Fukata S, Kuma K, et al. (2004) Transient hyperthyroidism after withdrawal of antithyroid drugs in patients with Graves' disease. Endocrin J 51: 213-217.

11. Suda H (2011) Current status and problems of endodontics in Japan. J Endod 32: 1-10. 